\title{
A dicotomia dos impactos causados pelo uso da internet em período de pandemia
}

\author{
The dichotomy of impacts caused by internet use in a pandemic period \\ La dicotomía de los impactos causados por el uso de internet en un período de pandemia
}

Recebido: 21/01/2022 | Revisado: 26/01/2022 | Aceito: 31/01/2022 | Publicado: 06/02/2022

Fernanda Abade Lemos

ORCID: https://orcid.org/0000-0001-9062-1270

Faculdade Irecê, Brasil

E-mail: fernandalemos222@outlook.com

Rodrigo Oliveira Damasceno

ORCID: https://orcid.org/0000-0002-0662-6827

Universidade Federal do Vale do São Francisco, Brasil

E-mail: digao.damasceno@yahoo.com.br

André Freire Silva

ORCID: https://orcid.org/0000-0002-8014-6661 Universidade Nove de Julho, Brasil

E-mail:andre.freire_silva@hotmail.com

Sabrina Andrade dos Santos

ORCID: https://orcid.org/0000-0003-4778-0852 Universidade Paulista, Brasil

E-mail: sabrinasantosandrade5@gmail.com

Uanderson Gomes dos Santos

ORCID: https://orcid.org/0000-0002-5641-8030

Faculdade Irecê, Brasil

E-mail: uandergomesz@outlook.com

\begin{abstract}
Resumo
Objetivo: compreender os impactos ocasionados pelo uso da internet em meio à pandemia da COVID-19. Métodos: trata-se de uma revisão sistemática da literatura de abordagem qualitativa e característica descritivo-exploratória realizada no ano de 2020. Resultados: existe uma dicotômica relacionada ao uso da internet em meio à pandemia da COVID-19. Por um lado sua utilização tem desencadeado sentimento de medo, ansiedade, angústia, tristeza, depressão e até mesmo cibercondria, em contrapartida, tem proporcionado tranquilidade, bem-estar mental e servido como estratégia de intervenção psicológica em plataformas online. Considerações Finais: destarte, faz-se mister disseminar informações a população para que possa estabelecer um manuseio seguro, moderado e equânime desse dispositivo, usufruindo beneficamente das possibilidades ofertadas a fim de fazer-lo um aliado.
\end{abstract}

Palavras-chave: Coronavírus; Mídias sociais; Internet; Saúde mental.

\begin{abstract}
Objective: to understand the impacts caused by the use of the internet in the midst of the COVID-19 pandemic. Methods: this is a systematic review of the literature with a qualitative approach and a descriptive-exploratory characteristic carried out in 2020. Results: there is a dichotomy related to the use of the internet in the midst of the pandemic of COVID-19. On the one hand, its use has triggered feelings of fear, anxiety, anguish, sadness, depression and even cyberchronicity, on the other hand, it has provided tranquility, mental well-being and has served as a psychological intervention strategy on online platforms. Final considerations: therefore, it is necessary to disseminate information to the population so that they can establish safe, moderate and equitable handling of this device, benefiting beneficially from the possibilities offered in order to make them an ally.
\end{abstract}

Keywords: Coronavirus; Social media; Internet; Mental health.

\section{Resumen}

Objetivo: comprender los impactos causados por el uso de internet en medio de la pandemia COVID-19. Métodos: se trata de una revisión sistemática de la literatura con enfoque cualitativo y característica descriptiva-exploratoria realizada en 2020. Resultados: existe una dicotomía relacionada con el uso de internet en medio de la pandemia de COVID-19. Por un lado, su uso ha desencadenado sentimientos de miedo, ansiedad, angustia, tristeza, depresión e incluso cibercronicidad, por otro lado, ha aportado tranquilidad, bienestar mental y ha servido como estrategia de intervención psicológica en plataformas online. Consideraciones finales: por lo tanto, es necesario difundir información a la población para que pueda establecer un manejo seguro, moderado y equitativo de este dispositivo, beneficiándose de las posibilidades que ofrece para convertirlo en un aliado.

Palabras clave: Coronavirus; Medios de comunicación sociales; Internet; Salud mental. 


\section{Introdução}

Uma luta incansável vem sendo travada por vários países contra um inimigo invisível a olho nu. Descoberto na cidade Chinesa de Wuhan em dezembro de 2019, um novo vírus passou a compor a família Coronaviridae, o SARS-CoV-2 (Severe Aute Respiratory Syndrome Coronavírus 2), agente etiológico da doença intitulada como COVID-19 (Corona Vírus Disease 2019) (Ahamed et 1., 2020). Classificada oficialmente como pandemia pela Organização Mundial de Saúde no dia 11 de março de 2020 devido ao aumento exponencial do número de casos global, a infecção provocada pelo SARS-CoV-2 ocorre por meio do contato com gotículas respiratórias de pessoas contaminadas podendo apresentar-se de forma assintomática ou não, conforme particularidades de cada organismo (WHO, 2020).

Assim, quando as manifestações se instalam variam desde um simples quadro gripal a sintomas respiratórios mais graves, destacando-se os idosos como o grupo de maior risco a sofrer consequências mais severas, principalmente aqueles que possuem um histórico de comorbidades preexistentes (Schmidt et al., 2020). De acordo com um estudo realizado pela WHOChina Joint Mission on Coronavirus Disease 2019 em mais de 50 mil casos confirmados, os sinais e sintomas característicos da COVID-19 são: febre, tosse seca e dispineia, podendo ocorrer também atralgia, congestão nasal e diarreia, dentre outros (WHO, 2020).

No dia 25 de fevereiro de 2020, o Brasil registrou o primeiro caso de COVID-19 no estado de São Paulo, a vítima diz respeito a um homem de 61 anos que havia retornado de uma viagem à Itália (Aquino, Silveira, Pescarini, Aquino, SouzaFilho, Rocha et al., 2020). Como consequência da sua alta taxa de transmissibilidade, pouco tempo depois da instalação do vírus, o país sofreu uma curva crescente e desenfreada superando o número de casos do país índice (China) com 30.425 casos confirmados e mais de 1.900 mortes registradas em 16 de abril de 2020 (Ministério da Saúde, 2020a). Esse fato extremamente preocupante repercutiu de forma internacional a ponto de o Imperial College em Londres, publicar um artigo intitulado "Estimating COVID-19 cases and reproduction number in Brazil" reforçando a importância em compreender a panorâmica epidemiológica desencadeada (Mellan et al., 2020).

Nesse viés, devido à rápida disseminação do vírus, medidas sociais de prevenção foram preconizadas pelo Ministério da Saúde como o uso de máscara facial, incentivo a higienização das mãos com água e sabão, utilização de álcool em gel, etiqueta respiratória e até mesmo distanciamento social, uma vez que, inexiste até o momento uma vacina eficaz conta essa doença (Ministério da Saúde, 2020b). Devido à desigualdade social existente no Brasil as medidas recomendadas não foram adotadas igualmente entre os diferentes grupos, contudo, milhões de pessoas se viram na obrigatoriedade de interromper as relações humanas face a face, e seguir a recomendação de ficar em casa, isolamento esse que potencializa e afeta a saúde mental da população mundial (Faro et al., 2020).

Em um estudo de revisão de literatura foi identificado os diversos impactos causados pela COVID-19 na saúde mental da população, apontando para sintomas como ansiedade, depressão, e estresse auto-relatado como respostas perante o vírus e com probabilidade de estar associado a distúrbios do sono (Rajkumar, 2020). Em outro estudo, também há o indicativo do surgimento dos efeitos psicológicos negativos como o estresse pós-traumático, pontuando que os efeitos negativos podem ser duradouros (Brooks et al., 2020).

Assim, pesquisas tanto de revisão bibliográfica quanto de entrevistas online relataram a existência de efeitos psicológicos negativos como o estresse pós-traumático, altos índices de ansiedade, depressão, uso abusivo do álcool e um menor índice de bem estar, pontuando que os efeitos negativos podem ser permanentes. Também foi encontrado que jovens entre 21 e 40 anos, possuem uma maior vulnerabilidade no que se refere à condição de saúde mental e uso do álcool (Ahamed et al., 2020; Brooks et al., 2020).

Destarte, a situação que a população mundial se encontra por conta da COVID-19 deixará consequências psicológicas e econômicas sem precedentes. Um dos fatores relacionados às questões psicológicas é o medo que por conta da pandemia tem 
o potencial de desencadear outros transtornos psiquiátricos como depressão, ansiedade de saúde, preocupação financeira, suporte social e solidão, sendo estes os sintomas originários do processo de isolamento social (Brooks et al., 2020; Pakpour, 2020; Tian et al., 2020)

Esse indicativo só deixa mais evidente a importância dos laços sociais e afetivos, como ações e comportamentos capazes de diminuir os efeitos negativos advindos da pandemia, sendo de suma importância que no período de quarentena, que a população seja mais altruísta na tentativa de minimizar tais impactos advindos do período de isolamento (Tiam et al., 2020).

É imprescindível frisar o quanto pandemia da COVID-19 vêm afetando a vida da população mundial, deixando-os em estado de alerta para sua gravidade. Isso pode ser percebido em países como a Itália, onde foi realizado um estudo e os resultados apontaram para uma alteração significativa na vida das pessoas, principalmente o sentimento de medo, onde esse medo de contrair o vírus tem aumentado os níveis de ansiedade da população (Pakpour, 2020). Além das alterações psicológicas identificadas como a ansiedade, foi percebido o aumento do índice de casos de depressão na população italiana apontado como um importante fator que afeta o ciclo do sono, conforme trás Casagrande e colaboradores (2020), causando assim distúrbios, e estão potencialmente relacionados com os problemas psicológicos descritos anteriormente (Ahamed et al., 2020; Rajkumar, 2020; Brooks et al., 2020).

Convenientemente, o panorama emergencial induziu o aumento das interações no mundo digital por meio do smartphone transformando-o no único meio de contato disponível mais utilizado, sendo a comunicação veiculada através da internet banda larga permitindo os usuários ter acesso a uma gama de informações sobre os últimos acontecimentos mundiais referente à COVID-19 (Cuan-Baltazar et al., 2020). Assim, o uso exagerado/anormal de meios de comunicação como o smartphone associado à internet, pode desencadear alguns transtornos, já que a população mundial vive em um período atípico com essa pandemia (Elhai et al., 2020).

Conseguintemente, com a hiperconectividade, a rápida disseminação de informações variadas a respeito das medidas protetivas (Lima et al., 2020), seja em formato de videoclipes, depoimentos, artigos de notícias, fotos, lives ou memes que retratam notícias sobre a pandemia, ao mesmo tempo em que os deixam informados pode gerar um potencial anciogênico e de medo global (Brooks et al., 2020). Isso dificulta a distinção entre notícias verdadeiras e fake news, e consequentemente a compreensão das pessoas em seguir corretamente as orientações expondo-as a atitudes inapropriadas e á riscos desnecessários (Deslandes \& Coutinho., 2020).

Assim, ao mesmo tempo em que o isolamento, o lockdawn e o distanciamento fazem-se medidas emergentes necessárias ao controle da pandemia, e que o uso da internet favorece simular uma situação de normalidade por meio de interações sociais e de trabalho, o tempo incerto de durabilidade dessas medidas atrelado ao uso excessivo da internet constituem-se como fator estressor para a saúde mental da população, podendo causar desde reações leves a transtornos graves (Deslandes \& Coutinho., 2020).

Destarte, tendo como objeto de estudo os impactos psicológicos, o objetivo norteador do presente estudo é compreender tais impactos ocasionados pelo uso da internet em meio à pandemia da COVID-19, sendo o interesse pelo estudo evidenciado pelo fato de ser um problema emergente e não compreendido em sua totalidade, com provisões ainda incertas no campo biopsicossocial.

\section{Métodos}

Trata-se de uma revisão sistemática da literatura de abordagem qualitativa e característica descritivo-exploratória realizada no ano de 2020. Esse tipo de revisão pode contribuir em reflexões sobre a pandemia da COVID-19, por se tratar de crise emergente, que demanda resposta rápida e efetiva. A linhagem característica do estudo possibilita descrever as diversas 
características do fato estudado utilizando para este fim uma variedade de informações e, por conseguinte, explorar todo conteúdo para obter maior familiaridade com a problemática a fim de torná-la mais entendível.

Para tal, foram utilizados os seguintes bancos de dados: PubMed/Medline (Medical Literature Analysis and Retrievel System Online), Scielo (Scientific Electronic Library Online), Periódicos da CAPS e Revistas Eletrônicas no Departamento de Ciências da Saúde. Foram empregues descritores como: Coronavírus. Mídias Sociais. Internet. Saúde Mental e Intervenções. Fora instituída a técnica de pesquisa bibliográfica para encontrar na literatura dados relacionados à problemática em questão em busca de respostas aos questionamentos por hora levantada nesse estudo, que por sua vez constitui-se da natureza básica.

Critérios de inclusão e exclusão foram previamente estabelecidos e a busca resultou em 89 artigos, destes apenas 29 se encaixaram na proposta do estudo, sendo 9 encontrados na base PubMed, 3 no Scielo, 6 nos periódicos da CAPS e 11 em Revistas Eletrônicas. Para inclusão foram utilizados artigos completos datados em 2020 nos idiomas português e inglês, que abordavam sobre a temática, excluindo textos em forma de resumo e resumo expandido.

A revisão acontecera entre o mês de agosto e setembro de 2020, buscando descrever melhor a dicotomia dos impactos causados pelo uso da internet em período de pandemia. De início realizou-se a busca que contemplasse a temática utilizando como suporte o DeCS - Descritores em Ciências da Saúde, e posteriormente foi empregue o método de leitura seletiva para coletar as informações pertinentes e coerentes com o tema. Sequencialmente ocorreu à revisão sistemática dos artigos, formulação de ideias e conclusão dos dados que foram interpretados buscando responder os questionamentos por ora levantados.

\section{Resultados e Discussão}

Em suma, o desenvolvimento do presente estudo possibilitou notar a existência de uma dicotômica relacionada ao uso da internet em período de pandemia, pois por um lado tem desencadeado sentimento de medo, ansiedade, angústia, tristeza, depressão e até mesmo cibercondria, em contra partida, tem proporcionado tranquilidade, bem-estar mental e servido como estratégia de intervenção psicológica em plataformas online. Assim, ao mesmo tempo em que possibilita ao usuário obter informações em tempo real sobre a crise emergente, expõe-no ao risco de adentrar no mundo digital das chamadas fake news notícias falsas, que podem provocar um impacto negativo na saúde mental em uma escala global (Cuan-Baltazar et al., 2020).

No século atual o mundo tem vivido em uma era digital existindo cerca de 2 bilhões de pessoas com acesso a internet que é a fonte pioneira em estabelecer conexões virtuais na modalidade online e a principal percussora na disseminação de informações (Cuan-Baltazar et al., 2020). Entretanto, as conexões estabelecidas nesse "mundo ilusório", seja por meio de plataformas como Instagram, whatsapp, Twitter, Facebook etc, dita a forma que o "eu" irá se exibir para os "outros", assim, em muitos momentos a imagem passada é apenas uma fachada que sobressai a realidade vivenciada, sendo também uma forma pretensiosa de o usuário se sentir "parte de algo", ser por hora aplaudido e aumentar sua auto-estima por meio de "likes" recebidos e aceitação nas mídias sociais (Deslandes, Coutinho, 2020).

O advento da internet permite que o usuário tenha o "mundo em suas mãos" com acesso a diversas informações que podem contribuir positivamente ou negativamente a depender da maneira e intensidade do seu uso. De acordo com o número de acessos alcançados por uma página em uma plataforma digital, sua posição se eleva aparecendo nos principais mecanismos de pesquisas aumentando a probabilidade de um indivíduo em visualizá-la (Cuan-Baltazar et al., 2020).

Assim, interligando os fatos à crise emergente, nota-se que as falsas notícias tem se espalhado em uma velocidade superior a do vírus e quando atrelada ao medo de ser infectado, a períodos prolongados de quarentena e isolamento, e perdas financeiras tem despertado sentimento de medo, ansiedade, irritabilidade, depressão e sintomas de estresse pós-traumático (Cuan-Baltazar et al., 2020; Deslandes \& Coutinho., 2020). 
Nesse sentido, a hiperconectividade torna-se uma característica intrínseca da sociedade digital, expondo o usuário ao risco de dependência. A instalação de uma pandemia provocada pelo SARS-CoV-2 aumentou a probabilidade dessa coletividade em sofrer transtornos psicológicos, devido às modificações do convívio social com o estabelecimento de medidas de isolamento atrelado à busca incessante por informações sobre a doença, gerando uma sobrecarga em seu processamento e afetando a saúde mental (Faro et al., 2020).

Um questionário transversal online com participação de 45.161 brasileiros, a saber, adultos e idosos, mostrou que $40,4 \%$ se sentiam tristes ou deprimidos, 52,6\% sentiam-se ansiosos ou nervosos, 43,5\% relataram o aparecimento de problemas do sono. Sendo assim, a saúde mental também merece destaque nesse cenário, uma vez que o bem-estar físico depende do bem-estar psicológico e quando este último está abalado se estabelece um desequilíbrio mental-corporal trazendo consigo várias consequências aos acometidos (Barros et al., 2020).

Ainda na linhagem dos impactos psicológicos, no que diz respeito ao país epicentro da disseminação viral, um estudo popular com 1.210 pessoas revelou que 53,8\% classificaram o impacto psicológico do surto como moderado ou grave, 16,5\% relataram sintomas depressivos moderados a graves, 28,8\% relataram sintomas de ansiedade e 8,1\% relataram níveis de estresse (Wang et al., 2020). Esses dados apontam a gravidade do impacto inicial provocado na saúde mental dos indivíduos, evidenciando a necessidade de um estudo aprofundado a fim de traçar em longo prazo e em escala real, os distúrbios permanentes ocasionados pela COVID-19, para então planejar um cuidado resolutivo na tentativa de minimizar os rastros deixados pelo tsunami SARS-CoV-2.

Desse modo, percebe-se que a interrupção da comunicação interpessoal pode ser o causador ou intensificador de transtornos psicológicos, transformando as interações digitais em uma válvula de escape. No contexto atual, a busca incansável por notícias em mídias sociais a respeito da pandemia e seus impactos, tem contribuído para o aumento da cibercondria, uma busca obsessiva online por informações referentes à saúde, geralmente sobre sintomas específicos, como consta em um estudo transversal realizado em participantes finlandeses, já que a sobrecarga de informação atrapalha em seu processamento final refletindo de forma negativa na saúde mental (Farooq et al., 2020; Starcevic et al., 2019).

A Diretoria de Comunicação da UERJ (2020), descreve uma pesquisa realizada em 23 estados brasileiros por meio de um questionário online com mais de 200 perguntas e participação de 1.460 internautas, evidenciou um aumento de $80 \%$ dos sentimentos de ansiedade e estresse, e um número dobrado dos casos de depressão que saltaram de 4,2\% para 8,0\%. A gravidade da situação é alarmante, uma vez que, casos de suicídios já formam constatados na Coréia do Sul e na Índia (Goyal et al., 2020).

Assim, pesquisas publicadas recentemente apontam para os altos níveis de transtornos psicológicos registrados causados pela pandemia da COVID-19, isso é um indicativo da necessidade de se conhecer melhor os impactos causados e a proporção do mesmo (Ahmed et al., 2020; Rajkumar, 2020; Brooks et al., 2020; Tian et al., 2020). O sentimento excessivo do medo de ser infectado, afeta o bem-estar biopsicossocial dos indivíduos predisponentes, em especial os profissionais de saúde que trabalham na linha de frente no combate ao Coronavírus, pois estão expostos ao alto risco de ser infectado e infectar outras pessoas, acarretando em frustração, preocupação, medo, culpa e ainda sobrecarga de trabalho (Deslandes \& Coutinho., 2020).

Nessa perspectiva, os estudos têm avançado no sentido de se compreender melhor as consequências psicológicas causadas por essa pandemia e identificaram que essa doença tem uma influência sócio-psicológica significativa na população. Esse dado é uma alerta para autoridades, pois ela indica a necessidade de se equipar os serviços de saúde psicológica como forma de prestar atendimento a pessoas que estão em alto risco de transtornos mentais (Aquino et al., 2020; Faro et al., 2020; Banerjee, 2020).

Ademais, o panorama geral do planeta se resume em pessoas em uso de máscaras, higienização das mãos sendo realizada frequentemente, antissepsia de objetos inanimados, circulação mínima de indivíduos nas ruas, distanciamento social, 
isolamento e lockdown. O sentimento de medo aflorado, pessoas apreensivas, agônicas, ansiosas, preocupadas e muitas incertezas sondando a mente humana, é esse o quadro global evidenciado pela pandemia da COVID-19.

A restrição ao direito básico do ser humano em se locomover foi necessária durante o enfrentamento dessa crise, sendo uma das medidas adotadas que tiveram resultado significativo no que diz respeito à diminuição da propagação da doença. Por outro lado, essa estratégia adotada a logo prazo trouxe consigo vários desafios a serem superados no campo da saúde mental, tanto para os acometidos quanto para os psicólogos no que concerne a restrições ao atendimento presencial, na maioria das situações (Ornell et al., 2020).

Nessa vertente, de acordo com o Conselho Federal de Psicologia, o suporte psicológico direcionado as pessoas em situação de urgência e emergência na pandemia tem sido sugerido por meio das tecnologias de informação e da comunicação, veiculados principalmente através da internet. No Brasil essa estratégia está sendo disponibilizada logo após realização do "Cadastro e-Psi", conforme consta na Resolução CFP no 4/2020 publicada em 26 de março de 2020, objetivando minimizar os impactos na área da saúde mental.

Assim, é notório que, ao mesmo tempo em que o uso da internet pode desestabilizar o indivíduo desencadeando o aparecimento de transtornos psicológicos é utilizada como um novo modelo de intervenção em meio a essa situação, estabelecendo, portanto, uma linha tênue entre o uso equilibrado e o uso abusivo dessa ferramenta (Casagrande et al., 2020; Lima et al., 2020; Ho et al., 2020). Assim, as verdadeiras informações devem ser transmitidas por veículos oficiais, objetivando minimizar impacto emocional negativo nas pessoas, e queda na visualização de fake news, objetivando reduzir o sentimento de ansiedade em relação à pandemia (Ho, Chee, Ho., 2020).

Nesse seguimento, conforme dados encontrados na literatura, outra orientação aos usuários é o cuidado com a exposição excessiva a informações, seja por meio de mídias digitais ou noticiários na televisão, lembrando-os sempre de checar a veracidade dessas informações. Na China, primeiro país a sofrer as consequências do SARS-CoV-2, uma das intervenções psicológicas contendo propostas psicoeducativas direcionadas a população foi o atendimento em plataformas online 24 horas por dia, além de disponibilizar materiais informativos e canais para escuta psicológica (Jiang et al., 2020).

O West China Hospital introduziu medidas tecnológicas através da internet veiculada pelo smartphone como uma intervenção de suporte utilizada por médicos, psiquiatras, psicólogos e assistentes sociais para com pacientes, seus familiares e equipe médica (Zhang et al., 2020). Assim, espera-se que a interação em plataformas digitais no contexto da pandemia possa contribuir na diminuição dos efeitos colaterais que o isolamento é capaz de provocar como ansiedade, depressão, solidão, propensão a tentativas de suicídios devido à falta de interação presencial e o clima de medo em relação às incertezas da pandemia (Deslandes \& Coutinho., 2020). Com o uso da internet também é possível estabelecer uma conexão com quem esta distante, especialmente os familiares e amigos, a fim de atualizá-los sobre sua situação e tranquilizá-los reduzindo de certa forma a sensação de isolamento, estresse e pânico.

No Brasil, os profissionais psicólogos colocaram-se a disposição para ajudar e prestar acolhimento as pessoas psicologicamente afetadas pela pandemia da COVID-19, em conjunto com outros profissionais de saúde que foram convocados pelo governo para prestar trabalho voluntário (Ministério da Saúde, 2020a). Enfatizando os trabalhadores da saúde mental, estes estão utilizando como alternativa, intervenções e atendimentos online, para darem continuidade ao desenvolvimento de suas atividades (Schmidt et al., 2020).

Ademais, as intervenções psicológicas devem seguir uma proposta dinâmica com foco inicial nos estressores relacionados à doença ou nos problemas de adaptação às limitações do período (Schmidt et al., 2020). Dessa forma, a organização das atividades diárias realizadas de forma segura, prática de exercícios físicos, cuidados com o sono, táticas de relaxamento e consolidação das conexões com a rede de apoio social tem sido descritas como estratégias para promover um bem-estar psicológico (Banerjee, 2020). 
Nessa continuidade, é imprescindível aumentar a conscientização da população-alvo sobre a patologia, com intuito de reduzir a ansiedade social e estimular mudanças comportamentais que poderão auxiliar no controle dessa enfermidade (WHO, 2020). Nota-se, que existem outros meios de intervenções que podem ser trabalhadas, todavia, a utilização de plataformas online faz-se usual, uma vez que promove um maior alcance do público alvo a ser tratado. Portanto, é imprescindível educar a população sobre o uso consciente e saudável das mídias sociais, pois pode ajudar a amenizar as consequências negativas observadas.

\section{Considerações Finais}

A crise emergente atual provocada pelo SARS-CoV-2 se instalou rapidamente a ponto de provocar uma pandemia em razão da sua alta taxa de infectividade, desencadeando problemas biopsicossociais revelando as fragilidades do sistema de saúde mundial em lidar com os desafios por ora encontrados. A quarentena o isolamento social e o lockdawn foram medidas estratégicas adotadas por vários países em diferentes momentos da crise objetivando diminuir o número de indivíduos infectados, entretanto, provocou um efeito rebote no que diz respeito aos impactos psicológicos desencadeados, tornando-se uma via de mão dupla. Isso não significa que tais medidas não devem ser usadas, todavia, tal merece destaque.

Nesse período a internet tornou-se a principal fonte disseminadora de informações a qual os usuários se conectaram, sendo fundamental na promoção do contato virtual com familiares e amigos distantes. Por outro lado, a hiperconectividade usada para minimizar a privação das conexões "face a face", atrelada à busca excessiva por informações referentes à saúde desencadeou vários problemas psicológicos, a saber, ansiedade, insônia, medo, depressão, transtorno de estresse póstraumático havendo relatos até mesmo de suicídio, fazendo-se essencial o desenvolvimento de intervenções no campo da saúde mental.

Dentre as estratégias psicológicas encontradas na literatura destacam-se: orientar a população a buscar informações verídicas por meio de veículos oficiais evitando deparar-se com fake news, organizar suas atividades diárias com segurança, praticar exercícios físicos, manter cuidados com o sono, realizar técnicas de relaxamento, evitar exposição excessiva a noticiários televisivos e mídias digitas, distribuição de cartilhas informativas e a principal intervenção descrita e mais viável no momento, já que a orientação é ficar em casa, é a busca de suporte psicológico online por meio das plataformas digitais disponíveis.

Desse modo, conclui-se que há uma dicotômica relacionada ao uso da internet em tempos de pandemia, pois, ao tempo em que esta pode desestabilizar o indivíduo desencadeando o aparecimento de transtornos psicológicos, é utilizada como um novo modelo de intervenção em meio a essa situação, destacando-se os efeitos entre o uso equilibrado e o uso abusivo dessa ferramenta. Destarte, faz-se mister estabelecer um manuseio seguro, moderado e equânime desse dispositivo usufruindo beneficamente das possibilidades ofertadas, a fim de fazer-lo um aliado, sendo necessário o desenvolvimento de estudos futuros sobre a temática.

\section{Referências}

Ahmed, M. Z., Ahmed, O., Aibao, Z., Hanbin, S., Siyu, L., \& Ahmad, A. (2020). Epidemic of COVID-19 in China and associated Psychological Problems. Asian Journal of Psychiatry, 51. 2020; 102092.

WHO. (2020). Coronavirus disease 2020 (COVID-19). Situation Report - 67. World Health Organization (WHO). https://www.who.int/emergencies/diseases/novel-coronavirus-2019/situation-reports

Schmidt, B., Crepaldi, M. A., Bolze, S. D. A., Neiva-Silva, L. \& Demenech, L. M. (2020). Saúde mental e intervenções psicológicas diante da pandemia do novo coronavírus (COVID-19). Estud. psicol. 37.

Aquino, E. M. L., Silveira, I. H., Pescarini, J. M., Aquino, R., Souza-Filho, J. A., \& Rocha, A. S., et al. (2020). Medidas de distanciamento social no controle da pandemia de COVID-19: potenciais impactos e desafios no Brasil. Ciênc. saúde coletiva [internet]. 2020 [citado em 05 Jun 2020]. 
Ministério da Saúde. (2020a). Portaria n 639, de 31 de março de 2020. Dispõe sobre a Ação Estratégica "O Brasil Conta Comigo - Profissionais da Saúde", voltada à capacitação e ao cadastramento de profissionais da área de saúde, para o enfrentamento à pandemia do coronavírus (COVID-19). Diário Oficial da União. [internet]. 2020.

Mellan, T. A., Hoeltgebaum, H. H., Mishra, W. C., Schnekenberg, R. P., \& Gandy, A. et al. (2020). COVID-19 cases and reproduction number in Brazil. Imperial College London [internet]. 2020 [citado em 8 May 2020].

Ministério da Saúde. (2020b). Máscaras caseiras podem ajudar na prevenção contra o coronavírus. Brasília: Ministério da Saúde [internet]. 2020 [citado 7 abr 2020].

Faro, A., Bahiano, M. A., Nakano, T. C., Reis, C., Silva, B. F. P., \& Vitti, L. S. (2020). COVID-19 e saúde mental: a emergência do cuidado. Estud. psicol. (Campinas) vol.37 Campinas 2020; Epub June 01.

Rajkumar, R. P. (2020). COVID-19 and mental health: A review of the existing literature. Asian Journal of Psychiatry. $52.2020 ; 102066$.

Brooks, S.K., Webster, R. K., Smith, L. E., Woodland, L., Wessely, S., Greenberg, N., \& Rubin, G. J. (2020). The psychological impact of quarantine and how to reduce it: rapid review of the evidence. The Lancet, 395, 2020; (10227), 912-920.

Pakpour, A. H. (2020). The fear of COVID-19 and its role in preventive behaviors. Journal of Concurrent Disorders. Vol. 2 No. 1. 2020; (58-63).

Tian, F. L. I. H., Tian, S., Yang, J., Shao, J., \& Tian, C. (2020). Psychological symptoms of ordinary Chinese citizens based on SCL-90 during the level I emergency response to COVID-19. Psychiatry Research 288. 2020; 112992.

Casagrande, M., Favieri, F., Tambelli, R., \& Forte, G. (2020). The enemy who sealed the world: Effects quarantine due to the COVID-19 on sleep quality, anxiety, and psychological distress in the Italian population. Sleep Medicine. 2020; S1389-9457(20)30213-6.

Cuan-Baltazar, J. Y., Muñoz-Perez, M. J., Robledo-Vega, C., Pérez-Zepeda, M. F., \& Soto-Vega, E. (2020). Misinformation of COVID-19 on the Internet: Infodemiology Study. JMIR Public Health Surveill. Apr-Jun, 2020; 6(2): e18444. doi: 10.2196/18444.

Elhai, J. D., Gallinari, E. F., Rozgonjuk, D., \& Yang, H. (2020). Depression, anxiety and fear of missing out as correlates of social, non-social and problematic smartphone use. Addictive Behaviors 2020; 105, 106335.

Lima, C. R. M., Sánchez-Tarragó, N., Moraes, D., Grings, L. \& Maia, M. R. (2020). Emergência de saúde pública global devido à pandemia COVID-19: desinformação, assimetria de informação e validação discursiva. Jornal Folha de Rosto, 2020.

Deslandes, S. F., \& Coutinho, T. (2020). O uso intensivo da internet por crianças e adolescentes no contexto da COVID-19 e os riscos para violências autoinflingidas. Ciênc. saúde coletiva [internet]. 2020 [citado em 05 Jun 2020].

Barros, M. B. A., Lima, M. G., Malta, D. C., Szwarcwald, C. L., Azevedo, R. C. S., \& Romero, D, et al. (2020). Relato de tristeza/depressão, nervosismo/ansiedade e problemas de sono na população adulta brasileira durante a pandemia de COVID-19. Epidemiol. Serv. Saúde, Ago $2020 ; 29$ (4) 24.

Wang, C., Pan, R., Wan, X., Tan, Y., Xu, L., Ho, C. S., \& Ho, R. C. (2020). Immediate psychological responses and associated factors during the initial stage of the 2019 coronavirus disease (COVID-19) epidemic among the general population in china. International Journal of Environmental Research and Public Health, 2020; 17(5), 1729.

Farooq, A., Laato, S., \& Islam, A., N. (2020). Impacto das informações online sobre a intenção de auto-isolamento durante a pandemia COVID-19: estudo transversal. J Med Internet Rev 2020; 22 (5): e 19128 DOI: $10.2196 / 19128$.

Starcevic, V., Baggio, S., Berle, D., Khazaal, Y., \& Viswasam, K. (2019). Cyberchondria and its Relationships with Related Constructs: a Network Analysis. Psychiatr Q [internet]. 2019 [citado em 6 may 2019]; 90 (3):491-505.

Diretoria de Comunicação da UERJ. (2020). Aumento de casos de depressão entre brasileiros durante a quarentena. Universidade do Estado do Rio de Janeiro. [internet]. 2020 [citado em 5 jul 2020].

Goyal, K., Chauhan, P., Chhikara, K., Gupta, P., \& Singh, M. P. (2020). Fear of COVID 2019: first suicidal case in India. Asian Journal of Psychiatry, [internet]. 2020 [citado em 1 mar 2020]; 49(101989).

Banerjee, D. (2020). The COVID-19 outbreak: crucial role the psychiatrists can play. Asian Journal of Psychiatry, $50.2020 ; 102014$.

Ornell, F., Schuc, J. B., Sordi, A. O., \& Kessler, F. H. P. (2020). "Pandemic fear" and COVID-19: mental health burden and strategies. Brazilian Journal of Psychiatry. [internet]. 2020 [citado em 3 abr 2020].

Conselho Federal de Psicologia. Resolução do exercício profissional nº4, de 26 de março de 2020. Dispõe sobre regulamentação de serviços psicológico prestado por meio de Tecnologia da Informação e da Comunicação durante a pandemia do COVID19.

Ho, C. S., Chee, C. Y., \& Ho, R. C. (2020). Mental Health Strategies to Combat the Psychological Impact of COVID-19 Beyond Paranoia and Panic. Annals of the Academy of Medicine 2020; v. 49, n. 1, p. 1.

Jiang, X., Deng, L., Zhu, Y., Ji, H., Tao, L., Liu, L., \& Ji, W. (2020). Psychological crisis intervention during the outbreak period of new coronavirus pneumonia from experience in Shanghai. Psychiatry Research, 2020; 286, 112903.

Zhang, C., Yang, L., Liu, S., Ma, S., Wang, Y., Cai, Z., \& Zhang, B. (2020). Survey of insomnia and related social psychological factors among medical staff involved in the 2019 novel coronavirus disease outbreak. Frontiers in Psychiatry. 2020; 11(306), 1-9. 